\title{
Editorial
}

\section{Diet and nutritional status during pregnancy}

Adequate nutritional status and good dietary intake during preconception and pregnancy have been recognized as major contributors to healthy birth outcomes. On the other hand, undernutrition and suboptimal diets with poor energy and micronutrients during pregnancy have been associated with poor fetal growth, pre-term delivery, poor infant survival and increased risk of chronic diseases in later life. Suboptimal prenatal diets have also been associated with gestational diabetes and pre-eclampsia in the mother and increased risk of stillbirth and largefor-gestational age in the baby.

While the global prevalence of low birth weight is declining slowly, the current prevalence of low birth weight is still estimated to be $15 \cdot 5 \%$ worldwide or more than 20 million infants worldwide ${ }^{(1)}$. Nearly $96 \%$ of these low-birth-weight babies are born in developing countries. One of the major goals in 'A World Fit for Children', the Declaration and Plan of Action adopted by the UN General Assembly Special Session on Children in 2002, was to reduce low birth weight incidence by at least one-third - by two-thirds by $2015^{(2)}$. And while some progress has been made, it has not been enough ${ }^{(3)}$. To achieve this goal, it is of paramount importance that women commence their pregnancies healthy and well nourished and that they continue good diets throughout pregnancy. In this issue we highlight ten articles focusing on pregnancy nutrition that span across continents and include low- to high-income countries.

\section{Nutritional adequacy during pregnancy}

In a review including sixty-two studies published from 1989 to 2011, Lee et al. ${ }^{(4)}$ report on energy and nutrient intakes of pregnant women in low- and middle-income countries. Overall, the ranges of mean/median intakes of energy, fat, protein and carbohydrate in Caribbean and Central/South American pregnant women were relatively higher than in women living in Africa and Asia. A large majority of the studies from Africa and Asia reported lower energy and macronutrient intakes by pregnant women than are recommended by the FAO/WHO. The percentage of energy intake from carbohydrate and fat varied inversely across regions, while protein as a percentage of energy intake was less variable. Almost all studies reported that $\mathrm{Fe}$ and folate intakes were below the Estimated Average Requirement (EAR), and most studies also reported that $\mathrm{Ca}$ and $\mathrm{Zn}$ intakes in pregnant women were lower than EAR or close to the EAR. While all studies in the Caribbean and Central/South America reported vitamin A intake higher than EAR, nearly half of the studies from Asia and more than half of the studies from Africa reported vitamin A intake below it. Worth mentioning is that all studies from the Caribbean and Central/South America were conducted among women in urban and periurban areas, while most of the studies from Asia and Africa were among women in rural/peri-urban or rural areas. Lee et al. conclude that the problems of unbalanced macronutrient profiles and multiple micronutrient deficiencies are common among pregnant women in developing countries across regions of the world, and that multidimensional intervention strategies are needed to improve the nutritional status of women during pregnancy.

Anaemia and Fe deficiency are a specific focus of several articles in this issue. Shamim et al. ${ }^{(5)}$ report an association of $\mathrm{Hb}$ concentration with plasma $\mathrm{Zn}$, vitamin $\mathrm{B}_{12}$, and $\alpha$ - and $\gamma$-tocopherol concentrations among 285 pregnant women with minimal Fe deficiency in rural north-western Bangladesh. In that study, about one in five women at median gestational age of 10 weeks were anaemic, with only $2 \cdot 1 \%$ having moderate to severe anaemia. The prevalence of $\mathrm{Fe}$, folate and vitamin A deficiencies was relatively rare $(1 \cdot 0 \%, 2 \cdot 8 \%$ and $7 \cdot 1 \%$, respectively), but $14 \cdot 7 \%$ had $Z$ n deficiency, $19 \cdot 7 \%$ had vitamin $B_{12}$ deficiency and nearly half $(43.5 \%)$ had $\alpha$-tocopherol deficiency. The findings emphasize the need for ensuring adequate micronutrients in the diet of the pregnant women, even in the context of Fe sufficiency, to reduce the risk of anaemia and associated adverse birth outcomes.

Habimana et al. ${ }^{(6)}$ report on iodine and Fe status in 225 pregnant women from rural, semi-urban and urban areas of Lubumbashi in the Democratic Republic of Congo. Over half of the women in their study had insufficient iodine intake based on WHO criteria for median urinary iodine concentration (UIC). Mean UIC was lower in late pregnancy when compared with early pregnancy, and the proportion of women with insufficient iodine intake also increased with gestational age. With respect to Fe deficiency, the prevalence was almost $40 \%$. Prevalence was highest in the third trimester of pregnancy and higher in rural than in semi-urban or urban areas. One in six pregnant women had both iron and iodine deficiency overall, but in rural areas it was as high as one in three women. Although that study did not examine other micronutrients, these findings suggest that in the Democratic Republic of Congo, as in many other developing countries, pregnant women have poor micronutrient intake and that Fe deficiency is often accompanied by 
deficiency in other micronutrient(s) as well. Clearly, the findings point the need for appropriate intervention strategies, with special attention to women in rural areas.

Fe deficiency is a problem in high-income countries, too. In their longitudinal study, Arija et $a l^{(7)}$ report that $16 \cdot 2 \%$ of 285 Spanish pregnant women were Fe deficient at gestational week 10 . At delivery, $46.0 \%$ of the women had developed Fe deficiency and $13.5 \%$ had developed Fe-deficiency anaemia. Women who had Fe deficiency at an early stage of pregnancy and did not take Fe supplements had significantly higher prevalence of Fe deficiency at delivery. Having no initial Fe deficiency and taking Fe supplements during pregnancy were found to have a significant protective effect against Fe deficiency at delivery, although moderate Fe supplementation (up to $\sim 60 \mathrm{mg} / \mathrm{d}$ ) did not prevent Fe-deficiency anaemia. The study revealed the fact that about half of Spanish pregnant women could be at risk of developing Fe deficiency in late pregnancy unless they have adequate Fe status at the beginning or take $\mathrm{Fe}$ supplements, thus emphasizing the nutritional need with respect to Fe for both pre-pregnant and pregnant women.

In another study from Spain, Rodríguez-Bernal et al. ${ }^{(8)}$ report on a wider variety of nutrients, and compliance with nutritional recommendations, in 822 pregnant women in their first trimester. Overall, more than half of the women did not meet recommended guidelines for cereal and legumes, carbohydrate, $n$ - 3 and $n$ - 6 fatty acids, while $71 \%$ of the women had higher total fat intake than recommended. Virtually all women had lower than recommended intakes of folate and vitamin $\mathrm{D}$, and nearly two-thirds of the women did not meet the recommended intakes for Fe and vitamin E. Analysis of contributory factors showed that younger and less educated women had lower intakes of protein and $n-3$ fatty acids, higher intake of trans-fatty acids and greater inadequacy for micronutrients overall. Further, compared with foreignborn women, women born in Spain had lower intakes of fruit and carbohydrate, but higher intakes of protein, total fat, SFA, MUFA and $n-3$ fatty acids. These findings suggest the importance of promoting a nutrient-rich and balanced diet for women of childbearing age before pregnancy and, further, of taking into account social and cultural factors in any intervention programme that is undertaken.

Supplements are a key part of strategies to promote nutritional adequacy in pregnancy. In a study of 7326 pregnant women in the USA, Sweden, Finland and Germany, Aronsson et al. ${ }^{(9)}$ report that over $90 \%$ of the women were using one or more types of supplement. Sixty-five per cent took vitamin D supplements, with the highest proportion ( $85 \%)$ of users in the USA. Overall, $16 \%$ of the women took fatty acid supplements, with the highest proportion in Germany (32\%), followed by the USA (24\%). Interestingly, in all countries, being pregnant with the first child was a strong predictor for taking any supplements. Maternal age less than 25 years, higher education level, BMI $\geq 25 \mathrm{~kg} / \mathrm{m}^{2}$ and smoking were also associated with supplement use in some countries. This multi-country study revealed that a large majority of pregnant women in industrialized countries use supplements. Understanding the various sociodemographic and behavioural factors associated with the use of supplements might be helpful in promoting supplement use in other populations and in other settings.

\section{Overnutrition and obesity}

At the other end of the spectrum is the problem of weight retention and obesity in pregnancy and postpartum. Excessive weight gain during pregnancy and failure to lose weight during the postpartum period are strong predictors of obesity among women of reproductive age. Further, maternal obesity influences birth outcomes as well as the health of the mother. In a study from southern Brazil, Drehmer et $a l .^{(10)}$ report a median postpartum maternal weight retention of $4 \cdot 4 \mathrm{~kg}$ among 370 women followed until 4-6 months postpartum. They found an 'unfavourable evolution' of BMI from pre-pregnancy to postpartum (which the authors term 'obesity risk') in $55 \cdot 1 \%$ of women. White bread consumption was the only food item associated with postpartum weight retention, and inadequate postpartum fibre intake increased obesity risk by $24 \%$. The findings are relevant when considering appropriate programmes for obesity control. Appropriate, low-cost dietary interventions are essential, and the problem is especially urgent in Brazil, where obesity is escalating as epidemic.

Tomedi et al. ${ }^{(11)}$ report an association between pre-pregnancy obesity and nutritional patterns of maternal micronutrient and essential fatty acid (EFA) status during midpregnancy among 129 US women. After adjusting for various confounding factors, obese women were more likely to be in the lowest tertile of EFA $(\mathrm{OR}=3 \cdot 0)$ and carotenoid $(\mathrm{OR}=4.5)$ dietary patterns than their normal-weight counterparts. Although obese women had lower mean concentration of plasma ascorbic acid and higher serum soluble transferrin receptors concentration, no association between obesity and micronutrient status was observed when values were adjusted for confounding factors. While the findings are important, better understanding of the consequences of lower EFA and carotenoid concentrations during pregnancy on fetal growth and development is necessary before considering any specific intervention for obese pregnant women.

\section{Dietary assessment in pregnancy}

Establishing the relationship of maternal dietary nutrient intakes with maternal and fetal outcomes requires accurate estimation of energy and nutrient intakes throughout pregnancy. Simple dietary techniques such as FFQ can help to identify specific nutrient deficiencies and are therefore important in developing appropriately targeted 
strategies to reduce the risk of adverse birth outcomes. In this issue we have included two papers on methodology of dietary assessment. One study from Brazil conducted by Barbieri et $a l^{(12)}$ reports on the relative validity of a quantitative FFQ against $24 \mathrm{~h}$ recall for estimating nutrient intakes over three trimesters among 103 pregnant women. The FFQ tool was found to be useful for assessing energy, lipids, protein, carbohydrate, fibre and a range of micronutrients such as $\mathrm{Fe}, \mathrm{K}, \mathrm{Zn}$, vitamin $\mathrm{B}_{6}$, riboflavin and niacin for the gestational period as a whole. About 33\% of the pregnant women were classified into the same quartile, another $71 \%$ into the same or adjacent quartiles and only $7 \cdot 8 \%$ into opposite quartiles according to the two different methods of assessing nutrient intakes. The FFQ provides one acceptable and useful method for assessing relative nutrient intake during pregnancy among women in Brazil. The other study, from the $\mathrm{UK}^{(13)}$, compares nutrient intakes assessed by FFQ with those obtained from food diary among severely obese pregnant women. Using the FFQ, $48.5 \%$ of obese and $47 \cdot 3 \%$ of lean women were correctly classified, while about $13 \%$ of obese and $10 \%$ of lean women were grossly misclassified. The average correlations for nutrient intake estimates between methods were 0.32 and 0.42 for obese and lean women, respectively. Given the rising prevalence of severe obesity in pregnancy, methods to assess intake in this population are much needed. While these findings indicate lower agreement in estimates for obese than for lean women, the FFQ may still serve as a reasonable option for assessing nutrient intake in severely obese pregnant women.

The collection of articles on nutrition and pregnancy in this issue of Public Health Nutrition demonstrates that a substantial proportion of women around the globe, across regions and national income categories, have poor nutritional status and/or poor diets, especially with respect to micronutrients that can have significant consequences on their children's birth outcomes and on their own health. Clearly, 'A World Fit for Children' begins with a world of fit mothers. Whether by monitoring and surveillance, dietary assessment methodology, epidemiology or intervention, the articles here are working towards the same goal, and there is still much to do.

Faruk Ahmed First Editor

Marilyn Tseng Editor-in-Chief

\section{References}

1. UNICEF/World Health Organization (2004) Low Birth Weight: Country, Regional and Global Estimate. New York and Geneva: UNICEF and WHO.

2. United Nations (2002) Report of the Ad Hoc Committee of the Whole of the twenty-seventh special session of the General Assembly General Assembly. Official Records Twenty-seventh special session Supplement No. 3 (A/S-27/19/ Rev.1). http://www.unicef.org/specialsession/documentation/ documents/A-S27-19-Rev1E.pdf (accessed May 2013).

3. UNICEF (2012) Ten years after the historic UN Special Session on children, UNICEF marks progress in creating 'A World Fit for Children'. http://www.unicef.org/policyanalysis/ index_62398.html (accessed May 2013).

4. Lee SE, Talegawkar SA, Merialdi M et al. (2013) Dietary intakes of women during pregnancy in low- and middleincome countries. Public Health Nutr 16, 1340-1353.

5. Shamim AA, Kabir A, Merrill RD et al. (2013) Plasma zinc, vitamin $\mathrm{B}_{12}$ and $\alpha$-tocopherol are positively and plasma $\gamma$-tocopherol is negatively associated with $\mathrm{Hb}$ concentration in early pregnancy in north-west Bangladesh. Public Health Nutr 16, 1354-1361.

6. Habimana L, Twite KE, Wallemacq P et al. (2013) Iodine and iron status of pregnant women in Lubumbashi, Democratic Republic of Congo. Public Health Nutr 16, 1362-1370.

7. Arija V, Ribot B \& Aranda N (2013) Prevalence of iron deficiency states and risk of haemoconcentration during pregnancy according to initial iron stores and iron supplementation. Public Health Nutr 16, 1371-1378.

8. Rodríguez-Bernal CL, Ramón R, Quiles J et al. (2012) Dietary intake in pregnant women in a Spanish Mediterranean area: as good as it is supposed to be? Public Health Nutr 16, 1379-1389.

9. Aronsson CA, Vehik K, Yang Y et al., on behalf of the TEDDY Study Group (2013) Use of dietary supplements in pregnant women in relation to sociodemographic factors a report from The Environmental Determinants of Diabetes in the Young (TEDDY) study. Public Health Nutr 16, 1390-1402.

10. Drehmer M, Camey SA, Nunes MA et al. (2012) Fibre intake and evolution of BMI: from pre-pregnancy to postpartum. Public Health Nutr 16, 1403-1413.

11. Tomedi LE, Chang CH, Newby PK et al. (2013) Prepregnancy obesity and maternal nutritional biomarker status during pregnancy: a factor analysis. Public Health Nutr 16, 1414-1418.

12. Barbieri P, Nishimura RY, Crivellenti LC et al. (2013) Relative validation of a quantitative FFQ for use in Brazilian pregnant women. Public Health Nutr 16, 1419-1426.

13. Mohd-Shukri NA, Bolton JL, Norman JE et al. (2012) Evaluation of an FFQ to assess total energy and nutrient intakes in severely obese pregnant women. Public Health Nutr 16, 1427-1435. 